\title{
In-House Globalization: The Role of Globally Distributed Design and Product Architecture on Product Development Performance
}

\author{
Bilal Gokpinar \\ Department of Management Science and Innovation, University College London, London, WC1E 6BT, UK, b.gokpinar@ucl.ac.uk \\ Wallace J. Hopp \\ Ross School of Business, University of Michigan, Ann Arbor, Michigan 48109, USA, whopp@umich.edu
}

Seyed M. R. Iravani

Department of Industrial Engineering and Management Sciences, Northwestern University, Evanston, Illinois 60208, USA iravani@iems.northwestern.edu

\begin{abstract}
C hanges in the global economy and technological advances are stimulating increased geographic distribution of new product design and development efforts. For large organizations that design and develop complex products, this geographic distribution has added a new layer of complexity to product development operations. In this empirical study of a large auto manufacturer, we examine the operational performance implications of splitting the design of vehicle subsystems across multiple geographic locations. Our results indicate that global distribution diminishes the chance of completing tasks on time and degrades subsystem design quality. Finally, by examining the interplay between subsystem centrality and global distribution, we found that higher centrality in the product architecture amplifies the impact of global distribution on subsystem error rates.
\end{abstract}

Key words: distributed work; global product development; networked design; product architecture

History: Received: January 2010; Accepted: October 2011 by Edward Anderson and Geoffrey Parker, after 2 revisions.

\section{Introduction and Literature Review}

Geographic distribution of knowledge-intensive work is a widespread phenomenon in the global economy. Companies are increasingly internationalizing their R\&D activites (Brockhoff 1998). Open source software such as Linux and Apache is being developed by programming communities that circle the globe (Fuggetta 2003). New product development (NPD) is following manufacturing in being outsourced to internal and external suppliers around the globe (Eppinger and Chitkara 2006). In a survey of 103 new product development firms, 54 had used or were using global teams for some of their NPD efforts (McDonough et al. 2001). The Wall Street Journal recently reported: "Ford Motor Co. is reorganizing its design and engineering centers in a move to make the company more global while speeding vehicle development. ... The engineering centers, located in different regions throughout the world, will be responsible for development of such components as engines and chassis." (Bennett 2008). But globalization of design processes also presents risks. For example, Boeing made a "quantum leap in farming out the design and manufacture of crucial components" (Hiltzik 2011) for its landmark Dreamliner 787 by collaborating with around 40 global partners on the majority of the aircraft's design (Stackpole 2007), but wound up "billions of dollars over-budget, and years behind schedule" (Ray 2011).

Nonetheless, there are several potential benefits from geographically distributed product development, including (i) reducing labor costs, (ii) acquiring technical expertise, (iii) gaining access to local markets by incorporating diverse customer values from different cultures, and (iv) increasing efficiency by conducting round-the-clock operation (Hinds and Kiesler 1998). Beyond these, two key trends have stimulated distributed NPD over the past two decades. One is political, with the opening of many new labor markets to the global economy (Johnston 1991). The other is technological, as CAD systems and collaborative project management software have made it possible to divide design work among distributed teams and coordinate their activities (Leonardi and Bailey 2008). 
Managing geographically distributed product development to achieve these benefits while avoiding the risks poses a number of questions about the decision to distribute NPD work, including the following: under what conditions and how should companies distribute their NPD work? Do the challenges and problems associated with globally distributed work offset the benefits? Which metrics should be used to evaluate the process of work distribution? These questions are particularly challenging for large organizations that develop large and complex products (e.g., airplanes, automobiles). Given their size and market reach, the critical decision for them is not whether to distribute the design work or not, but rather how to distribute this knowledge-intensive work in the most effective manner. This raises additional questions. Which parts of the product (e.g., architectural subsystems) should be distributed? Are some subsystems easier to manage and design across geographic boundaries? What is the role of architectural interdependencies in managing global design? In this study, we make use of a large archival data set of the engineering design system of a global auto manufacturer to provide empirical insights into these important questions.

Although it is a relatively new practice, scholars have begun to study distributed NPD from a variety of perspectives, including cross-border knowledge transfer (Subramaniam 2006), the role of team member dispersion on teamwork quality and performance (Hoegl et al. 2007), and the use of virtual global teams (Harvey and Griffith 2007). The traditional "make-buy" decision has been expanded to include product design as well as conventional production decisions (Ulrich and Ellison 2005). Other studies have considered the effect of learning in product design over time (Anderson and Parker 2002) and the role of uncertainty (i.e., market, creative, and process) in NPD planning (Anderson and Joglekar 2005). Although Baldwin and Clark (2000) suggested that outsourcing the components is beneficial to a firm due to increased competition among suppliers, Novak and Eppinger (2001) argued that when the designed product is highly complex, inhouse production is preferable due to coordination advantages. Parker and Anderson (2002) suggested that, although outsourcing significant portions of product development may provide competitive advantage, coordinating these outsourced parts could create new challenges in product development as well as in procurement along the supply chain. More recently, Anderson et al. (2007) outlined a number of specific impacts of distributed product development on the search, selection, transformation, and coordination processes involved in new product development.
Our work diverges from these studies in two major ways: first, instead of examining a high level makebuy decision, which is related to both design and production, we focus specifically on in-house engineering design within a large global organization. Although much attention has been given to outsourcing and its consequences, tactical issues resulting from globalization of in-house product development have received comparatively little attention (Krishnan and Loch 2005). Second, unlike most of the work in this stream, which treats critical decisions such as outsourcing or off-shoring as binary (e.g., either make or buy a part, either offshore a task or not), we consider partial offshoring of interconnected subsystems.

To study partial distribution of in-house engineering design, we must break products into their constituent parts. The literature on "modularity" does this. Modularity refers to "a special form of design which intentionally creates a high degree of independence or 'loose coupling' between component designs by standardizing component interface specifications" (Sanchez and Mahoney 1996). Researchers have pointed out the organizational benefits of product modularity in managing complex product interdependencies (Baldwin and Clark 2000, Schilling and Steensma 2001, Ulrich and Pearson 1998). Product modularity has also been examined from a knowledge-based view of the firm as a driver of modular organization structures. For example, by associating a modular organization structure with a modular product architecture, Sanchez and Mahoney (1996) used modularity as a framework for more effective knowledge management and strategic learning. Using a simulation study, Ethiraj and Levinthal (2004) examined the trade-offs between overly refined modularization and excess levels of integration. The most developed approach for matching the organization to a modular product structure is the Design Structure Matrix (DSM) methodology (Eppinger et al. 1994). In this study, we go beyond the qualitative analysis of DSM to quantify the level of interdependencies between architectural subsystems by using a network-based approach based on social network theory (Wasserman and Faust 1994). We then investigate both the direct effect of architectural interdependencies (i.e., position of a subsystem in the product architecture network) on the on-time performance of NPD work as well as its moderating effect on the relationship between global distribution of an architectural subsystem and the accuracy of NPD work. In a recent study (Gokpinar et al. 2010), we found that misalignment of organizational communication and product architecture is correlated with final product quality (i.e., warranty claims). In this study, we use the same data source but have collected entirely 
new data (i.e., for global vehicle programs rather than domestic vehicle programs) and have focused on different research questions pertaining to whether globalization introduces inefficiencies on global product design process. Consequently, while Gokpinar et al. (2010) focused on the product, this study is about the process. More specifically, in this study, we investigate the effects of in-house globally distributed design on two key process performance measures: process time (task delay) and process quality (subsystem error rates).

Much of the literature that deals with distributed work focuses on the organization rather than on the product. Issues such as culture, language, identity, conflict, and trust in geographically distributed teams have been examined in great detail in the organizations literature (Armstrong and Cole 1998, Hinds and Kiesler 1998, 2005, Jarvenpaa and Leidner 1999). Researchers have examined the role of geographic location on knowledge flow in organizations and have found that as the geographic distance between individuals increases, transmitting knowledge becomes more difficult (Allen 1977) or, equivalently, as the proximity increases, transmitting knowledge becomes easier (Saxenian 1994). Focusing on geographic distance within an organization, Hansen and Lovas (2004) showed that the negative effect of geographic distance (in the form of spatial, cultural, and national differentiation) can be offset by informal relationships of subsidiaries. At the organizational level, Bell and Zaheer (2007) differentiated between institutional and organizational ties and found that institutional-level ties act as knowledge transmitters if they are geographically proximate, whereas organizational ties do not show this property regardless of proximity. Our study contributes to the organizational literature on geographically distributed work by (i) introducing product architecture as a major factor in knowledge transmission across different geographic locations and (ii) empirically examining the moderating effect of product architecture on the relationship between global distribution of NPD tasks and performance.

From an operational standpoint, two key product performance metrics in all product development systems are speed and accuracy. The speed of the process matters because it affects time to market, while the accuracy of the process matters because it can impact speed (e.g., by avoiding task rework) as well as final product quality. Because, as we noted earlier in the context of the Boeing 787 program, global distribution of product development can potentially have a major impact on both speed and accuracy, researchers have begun to try to understand the nature of the impacts. For example, in an empirical study on globally distributed software development, Herbsleb and Mockus (2003) surveyed engineers on how often their work is delayed. They found that distributed work tasks take about two and a half times as long to complete as similar tasks where all the work is colocated. Also in a field study in geographically distributed software teams, Espinosa et al. (2007) addressed both speed and accuracy by studying time to complete an error-free project as a measure of team performance. For a discussion of development time and a list of other studies that use it as a performance measure, see Gerwin and Barrowman (2002). In this study, we focus on the automotive industry, in which the larger teams, longer development times, and physical components involved in vehicle development make the process quite different from software development. In our study, we address the issues of speed and accuracy via separate metrics. We consider speed by evaluating on-time compliance with task due dates. We consider accuracy by developing an error rate metric that compares the number of task errors to the total number of tasks.

In section 2, we develop the theoretical basis for our model and present our hypotheses. We then provide the details of our setting and describe our data set and research methodology in section 3. We present the models, analysis, and results of the empirical study in section 4, and we conclude with a discussion of our findings, their implications, and potential directions for further research in section 5 .

\section{Theory and Hypotheses}

Global distribution of work affects knowledge flow and performance within organizations through several mechanisms: first, there is a cost associated with coordinating activities in multiple locations. Geographic distance reduces informal and spontaneous conversation opportunities (Allen 1977) and conferences (Audretsch 2003, von Hippel 1994). While members of physically proximate teams often enjoy social similarity, shared values, and expectations (Latane et al. 1995), members of globally distributed teams may find it more difficult to establish such harmonious social environments (Kraut et al. 1998). Taken as a whole, the research in this literature suggests that, as the number of geographically distributed teams involved in a task increases, the increased difficulty of coordinating activities will tend to negatively impact the time to complete a task. Whether or not this tendency negatively affects due date performance depends on whether or not management properly accounts for the added complexity of coordinating a geographically distributed team when setting due dates. Because the effects are subtle and global design practices are new, we conjecture that 
managers are likely to be overly optimistic. This leads to our first hypothesis:

HyротнеSIs 1. All else being equal, the higher the number of global sites involved in a design task, the greater the chance of delay in the task relative to its due date.

This hypothesis has great managerial significance because of the importance of speed in product design. Failure to complete tasks on time can compromise the timeliness of product launches. Many studies have emphasized the importance of speed in the product development process (Stalk and Hout 1990). In addition to the well-publicized delays of the Dreamliner 787, which have cost Boeing billions, there are many examples of costly product launch delays. For example, delays of the Nintendo 3DS caused it to miss the 2010 holiday shopping season, resulting in a \$1.1 billion downgrade in estimates of annual profits by Nintendo (Soble 2010).

In addition to speed, accuracy is the other key measure of product development performance. Unless tasks are completed without errors, products will not meet design specifications. This is because (i) errors during the design process may lead to quality problems in the end product and/or (ii) corrective actions in response to errors mean additional costs and loss of valuable time for design engineers. Therefore, we consider the error rate (as defined in section 3.2) as a key performance measure. A design process with both low delay and low error rate is needed to bring high quality, competitively priced products to customers in a timely fashion.

An important way in which organizations avoid errors is through knowledge sharing (Cummings 2004, Haas 2006). For example, researchers have found that production networks (users, suppliers, manufacturers) with superior knowledge-sharing mechanisms out innovate firms with less effective knowledge-sharing mechanisms (von Hippel 1998), and investment in knowledge sharing routines between firms is a potential source of competitive advantage (Dyer and Singh 1998). However, studies have also found that transmission of knowledge within organizations is negatively affected by geographic distance between individuals (Allen 1977). In order to successfully perform their tasks within an organization, it is necessary for individuals to have both specific knowledge pertaining to their individual tasks and knowledge of other tasks with which their tasks interact. For example, in a product development environment, the designer of one component must know about the designs of other components with which his/her component has physical and/or logical interfaces. Geographic distance may hinder transfer of knowledge about related tasks and thereby lead to higher error rates for highly distributed subsystems.

Beyond knowledge transfer issues, research has shown that projects involving team members from different sites experience higher levels of conflict, which is detrimental to performance (Hinds and Bailey 2003). When accumulated over the many interdependent projects and tasks involved in developing a product, the impact of individual conflicts could be quite substantial. Therefore, we expect higher levels of conflict, and hence higher rates of error, to arise in subsystems that are more globally distributed than in those concentrated within a small number of sites.

Media richness is essential to the quality of communication (Daft and Lengel 1986). The three major communication means for product development activities, ordered by their media richness, are physical meetings, telephone calls, and electronic mail (Anderson et al. 2004). Geographic distance may reduce the likelihood of physical meetings, and language differences may reduce the likelihood of telephone calls (Anderson et al. 2007), both of which could lead to reduced media richness and hence lower communication quality. Furthermore, geographic distance not only reduces the communication frequency, but it also results in substitution of media-rich physical meetings by inferior telephone or email communications (Sosa et al. 2002). Consequently, reduced communication frequency and lower communication quality experienced in geographically distributed subsystems may lead to higher error rates in these subsystems.

Taken together, knowledge transmission, conflict problems, and communication issues can be expected to result in lower performance in globally distributed subsystems. Hence, we hypothesize the following.

HYPOTHESIS 2. All else being equal, the greater the global distribution of a subsystem, the higher the error rate for that subsystem.

Ulrich (1995) defines product architecture as "the scheme by which the function of a product is allocated to physical components" and argues that product architecture is important in managerial decision making because it is a key driver of a firm's performance. In contrast, a component is defined as a "physically distinct portion of the product that embodies a core design concept and performs a well-defined function" (Henderson and Clark 1990). Decoupling and independence of these components in the product architecture is at the heart of modularity (Baldwin and Clark 2000), which can facilitate significant flexibility in managing architectural complexities in both product development (Sanchez and Mahoney 1996, Schilling and Steensma 2001) and software development (MacCormack 2001, MacCormack et al. 2006). While a 
modularity perspective is helpful in conceptualizing architectural complexities, a complex networks approach is useful in quantifying and measuring these complexities.

For managerial purposes, many design organizations use the word "subsystem" to describe a subset of the product architecture which includes a group of components that collectively perform a higher level function. In a vehicle, examples of subsystems include "front suspension," "steering wheel," "door trim," "front seat," etc. These subsystems have many physical interfaces and functional dependencies among their constituent components. If we characterize subsystems as nodes of a network and link them according to the intensity of interdependencies between them, we can create a product architecture network. A subsystem with high centrality in the product architecture network is one that has many interdependencies with other subsystems.

Because the interdependencies between subsystems demand coordination and hence present opportunities for errors, we expect a subsystem which is heavily connected to other subsystems in the product architecture network to be more prone to potential design errors. In fact, in a recent study (Gokpinar et al. 2010), we observed that internal errors (i.e., design problems) tend to increase as subsystem centrality increases. But, because resources dedicated to these problems also increase with centrality and these resources can offset internal errors, we found an inverted U-shaped relationship between subsystem centrality and external errors (i.e., warranty claims). This leads us to expect a positive association between product network centrality and error rates. Thus, we conjecture:

Hypothesis 3. All else being equal, the higher the centrality of a subsystem in the product architecture network, the higher the error rate for that subsystem.

A successful product development project requires substantial collaboration among project group members, such as exchanging information and jointly solving design related problems (Brown and Eisenhardt 1995). Coordination of subsystems whose components are designed across multiple sites is more difficult due to increased geographic distance and knowledge transmission difficulties. We expect these coordination issues to have a deleterious effect on product development performance. We refer to this as the "coordination burden" imposed by geographic distribution of a task. On the other hand, when a firm distributes its product development efforts, it gains freedom in task assignments. In a manner similar to many strategic partnerships, offshoring, and outsourcing arrangements (Jarillo 1988, Metters 2008, Venkatraman 2004) in which firms capitalize on the expertise of specialists, we expect to see subsystems to be assigned to those locations with the highest competence (e.g., better technical and labor resources). If this is the case, then we should see a positive association between global distribution and product development performance. We call this the "specialization benefit." Therefore, our conjecture is that global distribution has two effects on the error rate: the "coordination burden," which increases the error rate, and the "specialization benefit," which reduces the error rate. We expect the coordination burden to increase in subsystem centrality, but we expect the specialization benefit to remain relatively constant in subsystem centrality. Hence, we conjecture that the "coordination burden" will dominate in high centrality subsystems and cause the error rate to increase in the global distribution score. However, in low centrality subsystems, we expect the "specialization benefit" to dominate and cause the error rate to decrease in the global distribution score. This leads to our final hypothesis:

HYPOTHESIS 4. Higher centrality in the product architecture network will amplify the relationship between global distribution score of subsystems and their error rates.

\section{Data and Model Development}

To test the above hypotheses, we conducted an empirical study of the vehicle development process of a global auto manufacturer. An automobile is a complex product made up of a large number of components, processes, and functions. The process of developing a new car involves thousands of people working on thousands of interdependent tasks for many months at multiple locations. These characteristics make the development of a new car an excellent area in which to study management of product development (Clark and Fujimoto 1991). Our study is based on a large archival database of "Engineering Change Orders (ECOs)." ECOs are "part of almost every development process" (Terwiesch and Loch 1999), and they are used extensively in industry to administer and document complex product development projects. Several researchers have described the central role of ECOs in product development efforts (Clark and Fujimoto 1991, Huang and Mak 1999, Loch and Terwiesch 1999).

We spent several months on site to observe and understand the NPD process and to collect and verify the necessary data. Fortunately, recent advances in computer technologies and data storage capabilities have allowed firms to manage and store large amounts of data related to the NPD process. To our knowledge, this study and that in Gokpinar et al. 
(2010) are the first to make use of an ECO database to study product development networks. This study is the first to do so in a globally distributed context.

As product design is an iterative process, many changes are made to parts, drawings, interfaces, etc. during the development of a vehicle. All of these iterations are captured via ECOs. An ECO may indicate a design related problem or mistake, but it may also indicate other issues and transactions as well. For example, other reasons for issuing ECOs include initial release of a part, changes in part specifications due to government regulations or cost-reduction initiatives, and styling changes in response to marketing initiatives. For each ECO, the reason for issuance is indicated by selecting the required reason code from a drop-down menu. There are approximately 40 reason codes in the system. In addition to the reason code, an ECO contains a rich set of data, including the location it was issued, engineers who are involved with it, part numbers associated with it, other parts that are affected by the change, and the targeted and actual dates of completion. Our client makes use of a computerized ECO system accessible by design engineers in all of its engineering design sites across the globe. This system facilitates formal communication and collaboration among design engineers at multiple locations.

To facilitate our analysis, we divided ECOs into three subsets according to their reason codes: (i) new release ECOs, which are issued for all parts of new models, including both new parts and re-used old parts with new part numbers, (ii) problematic ECOs, which indicate a design-related error, (iii) other ECOs, which include all other ECOs that are neither new release nor problematic (e.g., ECOs due to exogenous changes in government regulations or styling).

The data set we collected contains the entire set of ECO transactions for four global vehicle programs that were developed in multiple design centers around the world and launched in the 2006 model year. These ECOs were issued between 2002 and 2005. We point out that a vehicle program provides the platform for several models, which may be sold under different brand names in different countries. The four vehicle programs we investigated resulted in 11 new car models. For the four vehicle programs we studied, the ECO database included 28,540 ECOs that represented computerized records for almost 2,000 design engineers from 10 engineering design centers (all of which are located in different countries, including the United States, Canada, Mexico, England, and others) working on nearly 40,000 parts. These engineers were responsible for creating and modifying the parts, making sure they meet design specifications and coordinating interfaces with other parts.
To test our hypotheses, we analyzed this system at two different levels. First, to test Hypothesis 1 about the impact of global distribution on the on-time performance of NPD tasks, we studied the system at the level of individual tasks. Then, to test Hypotheses 2, 3 , and 4 , we studied the system at the level of architectural subsystems. We did this by following our client's practice of dividing the vehicle into 243 architectural subsystems, and then evaluating the impact of various factors on error rates in the development of these subsystems. We summarize our results below.

\subsection{On-Time Performance Model}

Because global design efforts involve tasks that are performed in multiple global locations, we first create a model to examine the relationship between global distribution of a task and its on-time performance. We regard each ECO as a design task, of which there are 28,540 in the four vehicle programs we studied. As we are interested in on-time performance at the task level, we develop our model at the ECO level. We do this by defining the following variables.

3.1.1. Dependent Variable: ECO Lateness. We measured on-time performance by using data from all types of ECOs. As we noted earlier, when an ECO is initiated by a design engineer, a target completion date is set by the product development managers. If it is completed on or before the due date, it is called on-time; if not, it is called late. ECO lateness is a key metric to assess the performance of NPD efforts at the task level. We measured lateness with a binary metric, which takes the value of 0 if the ECO is completed on time and 1 if it is late. We used this binary metric instead of the more conventional continuous definition of lateness (i.e., completion date minus due date) for two reasons. First, ECOs differ in terms of complexity, the number of people and parts involved, and other factors that could affect time to completion. Being one day late on a task that was allotted one week to complete is not comparable to being one day late on a task that was allotted one month to complete. Second, during our on-site observations, we noticed that for ECOs that were already late, some design engineers did not close them immediately when they were completed, but instead waited for several late ECOs to be completed and then closed them all together (presumably for paperwork economies of scale). This means that for some of the late ECOs, their closing date may not accurately reflect their actual completion date. Consequently, using a continous metric such "absolute lateness" or "percent lateness" could have biased our results. However, as engineers were evaluated on on-time performance and hence had substantial incentive to report completed ECOs as finished by their due date (i.e., while they might report a late ECO 
as later than it actually was, they would not report an on-time ECO as late), our binary lateness metric is not biased. Moreover, the fact that our client used it as a measure of timeliness gives us confidence that it is a metric of practical significance.

\subsubsection{Independent Variable: Number of Global}

Sites. As described in the previous section, when an ECO is issued, all related parts and engineers that will be affected by this change are listed in the ECO. An ECO cannot be completed before getting a sign off from all of the affected engineers, who may be spread over multiple sites. We define the number of global sites for an ECO as the total number of different locations of engineers listed on that ECO. Our client has 10 locations in 10 countries for engineering design, so this measure has a range from 1 to 10 .

3.1.3. Control Variables. Although our main variable of interest is the number of global sites associated with an ECO, other factors may influence on-time performance. Therefore, we control for the following factors.

Number of parts. This represents the total number of parts listed in an ECO, which may be an indicator for the complexity of the ECO task. Number of design engineers. This is the total number of design engineers listed on the ECO, which may negatively affect ontime performance due to communication and signoff delays. Planned duration of the ECO. This variable is calculated by subtracting the initiation date from the completion date. The planned duration of the project may be a proxy for the complexity of the task, or it may be the result of the high number of global sites. Nevertheless, we control for this potentially significant effect. for the originator country of the ECO by using $10-1=9$ indicator variables. Note that we do not examine specific pairs of countries, so we treat each different country as an individual and equal category.

Indicator for the vehicle program. Issuing an ECO and setting the due dates may also be influenced by the vehicle program it belongs to. Therefore we control for the vehicle programs using $4-1=3$ indicator variables.

Indicator for the ECO type. Type of the ECO could also have an impact on the likelihood of being late. So, we also control for ECO type (new release, problematic, other) by using $3-1=2$ dummies.

\subsection{Error-Rate Model}

We now turn from speed to accuracy. While we could evaluate on-time completion at the ECO level, it does not make sense to evaluate accuracy at the ECO level because of the coordination requirements between compnents and hence tasks. Hence, to evaluate process accuracy, we shift our level of analysis to the subsystem level. We develop our model by defining the following variables.

3.2.1. Dependent Variable: Error Rate. Design tasks are documented via ECOs. Hence, each ECO can be viewed as an opportunity for error. Ideally, each task should be completed correctly the first time. But because errors occur, corrective actions are required; these are documented as problematic ECOs. The fraction of ECOs for a subsystem that are problematic therefore constitutes an error rate, which serves as a measure of the accuracy (quality) of the design process for that subsystem. We define the error rate $^{1}$ for a subsystem as

$$
\text { Error rate }=\frac{\text { number of problematic ECOs }}{\text { number of new release ECOs }+ \text { number of problematic ECOs }+ \text { number of other ECOs }} .
$$

Indicator for the system management group. Our client divides a vehicle into six major system management groups (e.g., Chassis, Electrical, Powertrain). Each ECO is "owned" by one of these system management groups. As on-time performance may differ across groups, we controlled for it by using $6-1=5$ indicator variables. Note that in the analysis we used "Body" as the reference category, as it is the least specialized system in terms of the nature of tasks and consequently has the least variation in task times.

Indicator for the originator country. Although there are clear definitions and guidelines of the process, the practice of initiating an ECO and following it up may differ slightly across different locations, so we control
Note that, in the above equation, the number of problematic ECOs plus the number of new release ECOs plus the number of other ECOs is equal to the total number of ECOs.

3.2.2. Independent Variables: Product Architecture Network Centrality. We created a product architecture network at the subsystem level in the same manner as Gokpinar et al. (2010). Specifically, we defined each subsystem as a node and established weighted links between pairs of nodes by counting the number of co-appearances of two subsystems in the same new-release ECO. Note that we only made use of new-release ECOs to characterize architectural linkage of parts, because they are issued to initiate all 
parts for a new vehicle program and are not influenced by problems or exogenous changes. Because all parts that have a physical or a functional interface with a given part are listed in that part's new-release ECO, we can get a proxy for the strength of the architectural relationship between two subsystems by counting the number of times they have parts that are named in the same new-release ECO.

After creating the product architecture network, we calculated the degree centrality for each node (i.e., subsystem) by using UCINET 6 (Borgatti et al. 2002). This is computed as the sum of the weighted links emanating from the node. Degree centrality measures the strength of the architectural connectivity of a subsystem with the rest of the subsystems.

Global distribution score. We calculated the global distribution of each subsystem by making use of individual ECOs in that subsystem. As we described in Section 3.1, we can identify the number of global sites for each ECO. With these, we calculated the global distribution of a subsystem by calculating the weighted average of all ECOs in that subsystem. That is, if a subsystem has 100 ECOs, of which 20 are associated with one engineering design site, 30 are associated with two sites, 40 are associated with three sites, and 10 are associated with four sites, then the global distribution score for this subsystem is $[(20 \times 1)+$ $(30 \times 2)+(40 \times 3)+(10 \times 4)] / 100=2.4$.

3.2.3. Control Variables. Other factors that may be associated with subsystem error rate are the following:

Number of parts. As in the previous section, this represents the total number of parts in a subsystem, which may be a proxy for the size or complexity of the subsystem.

Fraction of new parts. This is the fraction of new parts (as opposed to reused parts from an older model) in a subsystem, which may a proxy for overall task difficulty in that subsystem.

Number of design engineers. As in the previous section, this is the total number of design engineers that are listed in the ECOs of a subsystem, which may be a proxy for the complexity of that subsystem.

Number of ECOs. This is the total number of ECOs in a subsystem, which could be an indicator of the size or complexity of a subsystem.

Fraction of late ECOs. This represents the fraction of ECOs associated with a subsystem that are not completed by their specified due date. This measure could be an indicator of overall task difficulty in a subsystem.

Indicator for vehicle program. Subsystem error rates may differ across different vehicle programs. So, we introduced three indicator variables for the four global vehicle programs we studied. ${ }^{2}$

\section{Analysis and Results}

In this section, we present our analyses of the above models and discuss the results. We use our on-time performance model (at the ECO level) to test Hypothesis 1 in section 4.1 and then test Hypotheses 2-4 using the error rate model (at the subsystem level) in section 4.2 .

\subsection{On-Time Performance Analysis}

Table 1 presents descriptive statistics and correlations for the ECO-level (i.e., task-level) variables. Note that about $17 \%$ of the ECOs in our data set were completed late. ECOs are associated on average with 2.87 global sites. The highest correlation is 0.236 $(p<0.001)$, which is between number of global sites and ECO lateness, and the relationship is in the predicted direction. This indicates that our estimation is unlikely to be affected by any serious multicollinearity problem.

Because the dependent variable (ECO lateness) is dichotomous (i.e., 1 if late, 0 if on-time), we used logistic regression, which is based on the maximum likelihood method. That is, our model predicts the likelihood of ECOs being late for completion. We first checked for multicollinearity by calculating variance inflation factors. They were all less than seven, which suggested that multicollinearity is not a serious threat to our analysis. We also analyzed residuals (studentized, the deviance residual, and the leverage) and concluded that our results are not biased by influential points.

Table 2 presents the results for a hierarchical logistic regression at the ECO level. We removed insignificant country indicators from the final model. The slopes estimated in a logistic regression are the natural $\log$ of the odds ratio, and, therefore, a positive logit indicates a positive association between the independent variable and the likelihood that the binary dependent variable equals one. The sign of the coefficient of our main variable of interest, the number of global sites, is positive and significant, which supports Hypothesis 1.

Model 1a provides the results with all variables except the number of global sites, while Model $1 \mathrm{~b}$ presents the final model after adding the number of global sites as an explanatory variable. The likelihood ratio test suggests that both models are significant at the $1 \%$ level $\left(\chi^{2}=32.65\right.$ and $\left.\chi^{2}=36.77, p<0.001\right)$. Also note that, when the number of global sites is included in Model $1 b$, the likelihood ratio $\chi^{2}$ increases from 41.16 to 45.53 . The increase between the $\chi^{2}$ values of the two 


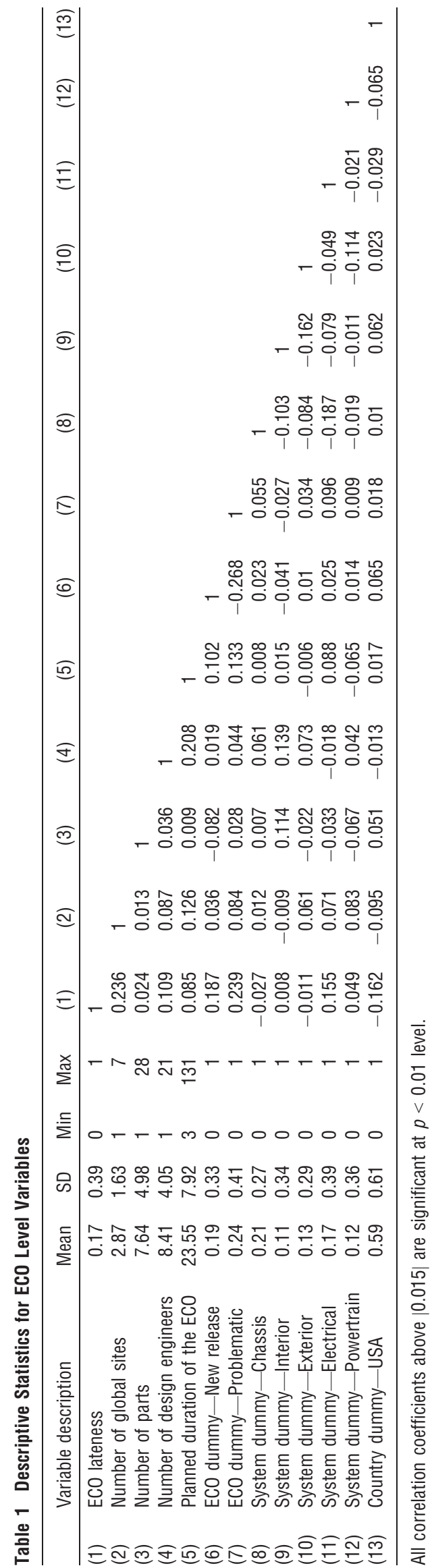

Table 2 Logit Model for ECO Lateness

\begin{tabular}{|c|c|c|}
\hline Variable & Model 1a & Model 1b \\
\hline Number of parts & $0.0055(0.0052)$ & $0.0051(0.0048)$ \\
\hline $\begin{array}{l}\text { Number of design } \\
\text { engineers }\end{array}$ & $0.0111^{\star \star *}(0.0052)$ & $0.0115^{\star \star}(0.0054)$ \\
\hline $\begin{array}{l}\text { Planned duration of } \\
\text { the ECO }\end{array}$ & $0.0092^{*}(0.0051)$ & $0.0088^{*}(0.0049)$ \\
\hline $\begin{array}{l}\text { ECO dummy- } \\
\text { New release }\end{array}$ & $0.2156^{\star \star \star}(0.0391)$ & $0.2109^{* * *}(0.0410)$ \\
\hline $\begin{array}{l}\text { ECO dummy- } \\
\text { Problematic }\end{array}$ & $0.3730^{\star \star \star}(0.0463)$ & $0.3752^{\star * \star}(0.0469)$ \\
\hline $\begin{array}{l}\text { System dummy- } \\
\text { Electrical }\end{array}$ & $0.4614^{* *}(0.2137)$ & $0.4599 * *(0.2130)$ \\
\hline $\begin{array}{l}\text { System dummy- } \\
\text { Powertrain }\end{array}$ & $0.2982^{*}(0.1704)$ & $0.2978 *(0.1700)$ \\
\hline System dummy_Chassis & $-0.1187(0.0848)$ & $-0.1208(0.0866)$ \\
\hline System dummy_-Interior & $0.0965(0.0803)$ & $0.0958(0.0819)$ \\
\hline System dummy_Exterior & $-0.1926(0.1750)$ & $-0.1937(0.1792)$ \\
\hline Country dummy - USA & $-0.4870^{\star * \star}(0.1691)$ & $-0.4867^{\star \star \star}(0.1682)$ \\
\hline Number of global sites & & $0.3237^{* * *}(0.0889)$ \\
\hline $\begin{array}{l}\text { Likelihood ratio } \\
\text { Chi-square }\end{array}$ & $41.16^{\star * *}(11 \mathrm{df})$ & $45.53^{* * *}(12 \mathrm{df})$ \\
\hline McFadden pseudo $\mathrm{R}^{2}$ & 0.172 & 0.191 \\
\hline$n$ & 28,540 & \\
\hline
\end{tabular}

models $(45.53-41.16=4.37)$ is also significant at the 0.05 level with $p=0.036<0.05$ ( $1 \mathrm{df})$, which indicates that adding the number of global sites improves the model significantly. Also, although the interpretation of the pseudo $R^{2}$ in a logit model is not the same as an OLS model (i.e., it does not explain the variation in the dependent variable), it is a widely used goodnessof-fit measure for logit models, and we observed an increase from 0.172 in Model 1a to 0.191 in Model 1b.

The simplest interpretation of Model $1 b$ is that, for every unit increase in the number of global sites, the odds of an ECO being late increases by a factor of $e^{0.3237}=1.382$ units (i.e., the odds ratio is equal to 1.382). While reporting odds is a common practice, this is not the most intuitive way to interpret the impact of globally distributed design in a probabilistic sense. Consequently, in order to better understand the relationship between the number of global sites and the likelihood of an ECO being late, we also examined the marginal effect of the number of global sites on ECO lateness, which varies with the value of all other explanatory variables. For each observation, as suggested by Hoetker (2007), we first calculated the response (i.e., ECO lateness) and then averaged these responses. We computed the marginal effect of the number of global sites on ECO lateness to be equal to $0.035(p<0.01)$. This implies that adding one site to the number of global sites would increase the probability of late completion of an ECO by $3.5 \%$.

Both the number of design engineers and planned duration of an ECO are significant and positively associated with the probability of a late ECO. This is not 
surprising, as both of these variables may be indicators of the complexity or difficulty of an ECO. Also, out of the six system indicator variables, only electrical and powertrain systems are significant and positively associated with ECO lateness relative to others. This is consistent with the intuition of senior design engineers, who suggested that these two systems are significantly more complex, and, hence, ECOs in these systems are more likely to be late than ECOs in the other systems, such as body or chassis. An interesting finding is that ECOs originated in the United States have a higher likelihood of being completed on time (i.e., as this variable is significant with a negative coefficient). ${ }^{3}$ This may be due to the fact that the ECO system has been employed in the United States longer than in other countries, so experience may have played a role. Also, as the United States is the main center for engineering design, with almost half of the entire global design operations, ECOs originated in the United States may have been resolved more efficiently by using the technical resources available at this location.

To ensure robustness of our findings, we investigated potential endogeneity issues and alternative explanations to our results at the task (ECO) level. For example, it can be argued that lateness of ECOs may increase the number of engineers or change the locations, rather than the reverse as argued in the study, which would constitute a reverse causation problem. We think that this form of endogeneity is not a serious threat here because of the unique process by which these ECOs are created and used in the design organization we studied. ECO's are created by design engineers, and a target completion date is set by product development managers. If an ECO is late, the typical managerial action is to first investigate the reason behind this lateness and then put some pressure on the people involved with that ECO (who are already listed in that ECO's distribution list). It is not practical to use additional people or change the location of an ECO once its lateness is realized. Such changes would only delay the completion of the ECO further. Consequently, the people associated with an ECO and the global sites associated with it are determined at the beginning of the ECO process (much earlier than a potential lateness is realized). This clearly rules out a reverse causation explanation of our results.

\subsection{Error Rate Analysis}

Table 3 provides the descriptive statistics and correlations for the subsystem-level variables. The highest correlation between any of the two explanatory variables is 0.295 , which is between the number of design engineers and the number of ECOs. Again, this suggests that multicollinearity should not be a problem. We note that both global distribution score and product network centrality have positive correlations with the error rate, as expected. In order to examine the association between these variables and error rate, we constructed a regression model. We analyzed the studentized residuals to examine outliers, which did not indicate any problems. We also tested for multicollinearity by calculating variance inflation factors (VIF's) for each of the variables. We found that they are all less than four, which confirms that multicollinearity is not a threat to our analyses. We also checked for heteroskedasticity in two ways. First, we examined the plot of residuals vs. independent variables. We observed that residuals are roughly similar in terms of size (width), which suggests that there is no serious heteroskedasticity threat. Next, we performed White's test (White 1980) and calculated the chi-squared statistic to be 7.32 , which is smaller than $\chi^{2}(6)=12.59$, so the homoskedasticity assumption is not rejected.

We exploited the panel structure of our data set by making use of the four global vehicle programs we studied. Specifically, because each vehicle program has 243 subsystems, we were able to observe the cross-section of these 243 subsystems four times (one observation per vehicle program). Considering the panel structure of the data, our hypotheses could be tested by creating either a random-effects or a fixedeffects regression model. A random-effects model assumes that the individual effects are uncorrelated with the regressors and that the individual specific constant terms are randomly distributed across crosssectional units. This greatly reduces the number of

Table 3 Descriptive Statistics for Subsystem Level Variables

\begin{tabular}{|c|c|c|c|c|c|c|c|c|c|c|c|c|c|}
\hline & Variable description & Mean & SD & Min & $\operatorname{Max}$ & (1) & (2) & (3) & (4) & (5) & (6) & (7) & (8) \\
\hline (1) & Error rate & 0.345 & 0.128 & 0.092 & 0.705 & 1 & & & & & & & \\
\hline (2) & Product network centrality & 0.186 & 0.159 & 0.084 & 0.588 & 0.093 & 1 & & & & & & \\
\hline (3) & Global distribution score & 2.961 & 0.873 & 1.38 & 5.93 & 0.156 & 0.071 & 1 & & & & & \\
\hline (4) & Number of parts & 208.7 & 16.41 & 136 & 318 & -0.002 & 0.013 & 0.008 & 1 & & & & \\
\hline (5) & Fraction of new parts & 0.319 & 0.184 & 0.098 & 0.625 & 0.297 & 0.014 & 0.011 & -0.003 & 1 & & & \\
\hline (6) & Number of design engineers & 136.7 & 34.074 & 39 & 306 & -0.005 & -0.028 & 0.185 & 0.005 & -0.021 & 1 & & \\
\hline (7) & Number of ECOs & 265.9 & 25.76 & 108 & 384 & 0.082 & 0.169 & 0.083 & -0.016 & 0.142 & 0.295 & 1 & \\
\hline (8) & Fraction of late ECOs & 0.198 & 0.204 & 0.045 & 0.388 & -0.181 & 0.107 & 0.215 & 0.010 & 0.092 & -0.119 & 0.008 & 1 \\
\hline
\end{tabular}

All correlation coefficients above $|0.08|$ are significant at $p<0.01$ level. 


\section{Table 4 Random-Effects Model for Error Rate}

\begin{tabular}{|c|c|c|c|c|c|}
\hline Variable & Model 2a & Model 2b & Model 2c & Model 2d & Model 2e \\
\hline Number of parts & $0.002(0.012)$ & $0.003(0.014)$ & $0.003(0.013)$ & $0.003(0.013)$ & $0.003(0.014)$ \\
\hline Fraction of new parts & $0.788^{* *}(0.342)$ & $0.790^{* *}(0.346)$ & $0.782^{\star *}(0.344)$ & $0.788^{\star *}(0.345)$ & $0.785^{\star \star}(0.347)$ \\
\hline Number of design engineers & $-0.004^{*}(0.002)$ & $-0.003^{\star}(0.002)$ & $-0.004^{*}(0.002)$ & $-0.004^{*}(0.002)$ & $-0.004^{*}(0.002)$ \\
\hline Number of ECOs & $0.002(0.011)$ & $0.002(0.010)$ & $0.003(0.008)$ & $0.002(0.009)$ & $0.003(0.007)$ \\
\hline Fraction of late ECOs & $-0.348^{* \star}(0.166)$ & $-0.361^{\star *}(0.181)$ & $-0.368^{*}(0.195)$ & $-0.352^{* *}(0.0176)$ & $-0.373^{*}(0.199)$ \\
\hline Product network centrality & & $0.185^{\star *}(0.077)$ & & $0.181^{\star *}(0.076)$ & $0.176^{\star \star}(0.083)$ \\
\hline Global distribution score & & & $0.042^{\star * *}(0.013)$ & $0.040^{\star * *}(0.014)$ & $0.039^{* \star *}(0.014)$ \\
\hline $\begin{array}{l}\text { Product network centrality } \times \text { global } \\
\text { distribution }\end{array}$ & & & & & $0.114^{\star * *}(0.041)$ \\
\hline$F$ statistic for the partial $F$-test & & $82.41 * * *$ & $122.95 * * *$ & $7.837 * * *$ & $7.893 * * *$ \\
\hline Adjusted $R^{2}$ & $29.7 \%$ & $35.2 \%$ & $37.6 \%$ & $38.3 \%$ & $38.9 \%$ \\
\hline$n$ & 972 & & & & \\
\hline
\end{tabular}

${ }^{\star} p<0.1,{ }^{* *} p<0.05,{ }^{* * *} p<0.01$.

parameters to be estimated, which makes it appropriate for data sets with large cross sections and small time units (i.e., repeated observations) (Greene 2008). In contrast, a fixed-effects model specifically accounts for the omitted effects which are correlated with the included variables. However, the fixed-effects model is costly in terms of degrees of freedom lost, as it introduces fixed effects for each cross-sectional unit.

Because our data set included a large cross section of observations (i.e., 243 subsystems), with limited time units (i.e., only four vehicle programs), a random-effects model is best suited for our purposes. We confirmed this with the Hausman specification test (Hausman 1978) of the choice between a randomeffects and a fixed-effects specification, and resulted in a test statistic of $\chi^{2}=6.85$, which is not significant at the 0.05 level $(p>0.05)$. Therefore, we do not reject the null hypothesis, which was that random-effects and fixed-effects estimates do not differ significantly. Consequently, we picked the more efficient and consistent random-effects model over the fixed-effects model and used generalized least squares (GLS) to analyze it.

Table 4 provides the results of the hierarchical random-effects model for error rates. Model 2a includes all base variables, while Models $2 b$ and $2 c$ add the product network centrality and global distribution score separately. Finally, Model 2e includes all variables including the interaction of product network centrality and global distribution score.

Model $2 \mathrm{~b}$ indicates that product network centrality is significant $(\beta=0.185, p<0.05)$ and has a positive impact on the error rate. Moreover, a partial $F$ test indicates that Model $2 \mathrm{~b}$ provides significant improvement over Model 2a for predicting the error rates. This provides support for Hypothesis 2 that higher subsystem centrality is associated with higher error rates. The global distribution score is also significant $(p<0.01)$ in Model 2c, which supports Hypothesis 3. Also, the partial $F$ statistic is $122.95(p<0.01)$, which suggests that adding the global distribution score improved the fit from Model 2a to 2c. The implication is that as subsystems get more global, the error rate increases significantly. Next, Model 2d includes both product network centrality and global distribution score. Both variables are again significant at $p<0.05$ and $p<0.01$ levels, respectively, and the partial $F$ test indicates a significant improvement with the addition of both variables (Model 2d) over Model 2c.

Finally, in the presence of both the direct effects of product network centrality and global distribution score, the interaction term product network centrality $\times$ global distribution score is also significant at the $p<0.01$ level, which provides evidence for Hypothesis 4 . The partial $F$ statistic between Model 2d and Model 2e is calculated to be $7.893(p<0.01)$, which suggests that adding the interaction term has improved the explanatory power of the model significantly. This supports Hypothesis 4 that product network centrality amplifies the relationship between global distribution score and error rates.

To further examine the conjectured amplifying effect, we plotted the interaction between product network centrality and global distribution score in Figure 1. Using our sample, we identified the values of one

Figure 1 The Impact of Subsystem Centrality on the Relationship Between Global Distribution Score and Error Rate

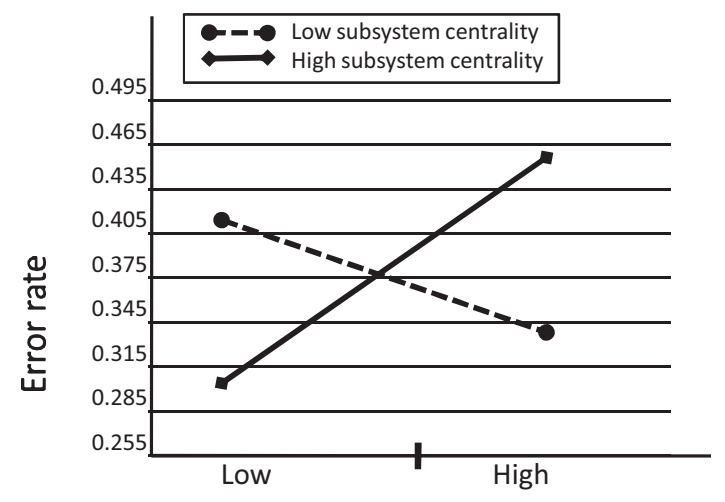

Global distribution score 
standard deviation above and below of the means of subsystem centrality and global distribution (i.e., four points with high centrality and high distribution, high centrality and low distribution, low centrality and high distribution, low centrality and low distribution) and obtained the corresponding error rates. We then plotted these in Figure 1 to visually confirm the interaction effect. Consistent with Hypothesis 4 and the results of Model 2e, there is a strong positive relationship between global distribution score and error rates when the subsystem has high product network centrality. That is, when highly central subsystems are distributed globally, they experience higher error rates. In contrast, we observe a slightly negative relationship between global distribution score and error rates for low centrality subsystems. That is, when low centrality subsystems are distributed globally, they experience lower error rates. This is consistent with our conjecture that the "coordination burden" dominates for highly central subsystems, so error rate increases as the global distribution score increases. In contrast, the "specialization benefit" dominates for low centrality subsystems, so the error rate decreases as the global distribution score increases.

Model 4 also reveals that the fraction of new parts is a significant predictor of the error rates of the subsystems. This is not surprising, as new parts imply a learning curve and hence more errors. Also, the fraction of new parts may be a good indicator of task difficulty within the subsystems. Interestingly, the number of design engineers is significant but with a negative coefficient. That is, error rate in a subsystem decreases as the number of design engineers increases. We conjecture that this is a consequence of more engineers in a subsystem providing more pairs of eyes to catch design errors. But our results also imply that this reduction in error rate comes with a price in terms of task delay. That is, the positive association of number of design engineers and probability of task delay in Table 2 suggests that more engineers lead to higher task delay. Finally, the fraction of late ECOs has a negative and significant coefficient. This indicates that, if engineers in a subsystem are not pressured to complete design tasks on time (resulting in a higher fraction of late ECOs), they may be able to achieve a lower rate of errors. In short, our data suggest a trade-off between higher on-time performance and lower error rates.

To examine the robustness of our results, we examined potential endogeneity issues in our analysis. First, as with any random-effects model, if the unobserved individual heterogeneity is correlated with the explanatory variables, this may introduce an endogeneity problem and therefore lead to potentially inconsistent estimates. To check this, we created a fixedeffects model in which we introduced a dummy variable for each subsystem. This enabled us to better control for any unobserved subsystem-specific characteristics. We examined the model coefficients of this fixed-effects regression and found that all estimates are within the $10 \%$ confidence interval of our random-effects estimates, and all significant estimates of the random-effects model are still significant at the same levels. Therefore, the fixed-effects specification does not change our results. While this fixed-effects specification alleviates the major concerns regarding endogeneity, it does not rule it out entirely. For example, one could argue that a third factor such as complexity could affect both subsystem centrality and error rates, or reverse causality could be a concern as higher error rates might lead to greater global distribution of a subsystem. Ideally, the first concern (i.e., a third factor affecting both centrality and error rates) could have beeen addressed via a two stage least squares (2SLS) estimate using instrumental variables. Unfortunately, we were unable to find strong and suitable instruments to use in a 2SLS regression in our data. So, instead, in our model, we controlled for a variety of factors such as the number of parts, the number of design engineers, and the number of ECOs that might act as proxies for subsystem complexity. We also controlled for individual subsystem characteristics (by introducing dummies) in our additional fixed-effects regression. Finally, we can rule out potential reverse causality between global distribution and error rates because the distribution of an ECO is determined at the beginning of the process, whereas errors occur only after engineers start working on their parts. So, global distribution clearly precedes errors.

To get a sense of the economic significance of our main explanatory variables, we changed their values by one standard deviation and calculated the associated effect on error rate. A one standard deviation increase in product network centrality results in a $100 \times(0.159 \times 0.176) / 0.345=8.1 \%$ increase in error rate. Similarly, a one standard deviation increase in the global distribution score results in a $100 \times$ $(0.873 \times 0.039) / 0.345=9.8 \%$ increase in error rate These effects are quite substantial considering the waste and cost associated with making design related errors.

\section{Discussion and Conclusion}

The impact of globally distributed work and the role of geographic distance on knowledge transmission have been studied by management scholars. Most of these earlier studies have focused on the behavioral and cultural characteristics of distributed work and their implications. This study contributes to our understanding of global product development by examining the operational impact of global distribu- 
tion on speed and accuracy of the design process. We found that increasing the degree of global distribution of a design task makes it less likely to be completed on time. Specifically, our results indicate that globalization leads to missed due dates, not just longer task times, which suggests that management tends to underestimate the impact of globalization on development times when setting due dates. Although one of the reasons for companies to engage in global design activities is to improve operational efficiency, our results suggest that this may not be the case. If a design task is distributed among several global locations, coordination is likely to be a key challenge even in the presence of computerized systems designed to facilitate communication and collaboration. Even after controlling for the number of design engineers involved and the subsystem associated with the task, we observed that there is a positive association between the number of global sites involved and the chance of an ECO being late.

We also examined the role of product architecture and global distribution on the performance of engineering design efforts at the subsystem level. Our results suggest that the location of a subsystem in the product architecture network may affect the quality of the design work in that subsystem. As a subsystem becomes more central in the network (i.e., it involves more interfaces and dependencies with other subsystems), the error rate in that subsystem increases substantially. Our study provides an innovative way to measure the strength of these interdependencies via standard data from an engineering change order system. As such, it may help managers to assess some of complex interdependencies present in their product architecture and the associated risks of global distribution of design activities.

Our results regarding the role of global distribution of subsystems implies that global distribution could be detrimental to design quality. After controlling for such factors as fraction of new parts or the number of design engineers, we found that increasing the global distribution of a subsystem leads to higher error rates. Moreover, we found that central subsystems in the product network are particularly vulnerable to this negative effect of global distribution. Spreading central subsystems across multiple design locations is likely to lead to high rates of errors. As these errors increase cost (i.e., a part with a design mistake must be re-done) and have the potential to cause delay in product launches, product development managers should keep a close eye on subsystems that are highly central and globally distributed. Indeed, it may make sense not to globally distribute subsystems with high centrality and instead assign their design to a single location or divide their design among only a few locations. This type of concentrated design assignment may help the product development organization better coordinate the many interdependent tasks associated with highly central subsystems.

Although distributed design has received considerable attention in recent years, our understanding of some forms of distributed organizational arrangements and their performance implications are limited. Research in organizational arrangements such as outsourcing or off-shoring is relatively well developed, but the phenomenon of in-house globalization has not yet received much attention. Integration of knowledge-intensive operations across multiple global locations and management of these operations within a firm are significant challenges that call for further study by scholars from multiple disciplines. Using a unique data set and innovative metrics, we have empirically tested the impact of product architecture and global distribution on design performance. As such, our study extends the knowledge of distributed design efforts and provides conceptual insights into globalized in-house design. Our work is also of practical relevance to product development managers, as we make use of a standard database that is readily available to many product development organizations.

Our work points to several potential directions for future research. We examined the role of product architecture and global distribution on product development performance. The impact of these variables on end product quality, as well as the interplay between these product design metrics and manufacturing, could be investigated. Also, we only considered development efforts for one model year, due to data limitations. A longitudinal study that captures the dynamic nature of product design efforts and monitors the improvements over time could reveal the sensitivity of performance to shifts in the degree of product distribution. Another obvious direction for future research is complementing this study with more detailed ethnographic studies of distributed work. This would facilitate investigation of informal communication practices of design engineers and the interplay between geographic location and product architecture on informal communication and performance via a survey instrument. Another avenue for study could be to examine how coordination across different pairs of global locations vary (e.g., coordinating between the United States and Canada vs. the United States and India) and how this might affect product development performance. Finally, like many empirical studies, this work primarily relies on data from one company in a specific industry (i.e., automotive); it remains to be seen whether our results extend to other settings and industries. 


\section{Acknowledgments}

The authors would like to thank the special issue editors and two anonymous reviewers for many helpful comments and suggestions. The authors gratefully acknowledge the support of this research by the National Science Foundation under Grants DMI-0423048 and DMI-024377.

\section{Notes}

${ }^{1}$ Note that the independent variable "fraction of problematic ECOs" in Gokpinar et al. (2010) is identical to the dependent variable "error rate" here. We use the term "error rate" to better convey the role of the variable in our model.

${ }^{2}$ In subsequent analysis, we found that there is no difference between vehicle programs in their effect on subsystem error rates. Therefore, for parsimony, we did not include these dummy variables in our final regression.

${ }^{3}$ As we have 10 countries, we used nine dummies and one reference country. We used stepwise regressions and several different model specifications such as having the United States as the reference category, or Mexico or Canada as the reference. We observed that when the United States is the reference, although their coefficients are quite small, all of the country dummies were positive and significant. But when we used one of the other countries as the reference, only the United States had a significant and negative coefficient. So, we only included the United States in our final model, and we concluded that ECOs originated in the United States are more likely to be on time relative to those originated in other countries.

\section{References}

Allen, T. J. 1977. Managing the Flow of Technology. MIT Press, Cambridge, MA.

Anderson, E., A. Davis-Blake, S. Erzurumlu, N. Joglekar, G. Parker. 2007. The effects of outsourcing, offshoring, and distributed product development organization on coordinating the NPD process. S. Kavadias, C. Loch, eds. Handbook of New Product Development. Elsevier/Butterworth-Heinemann, Burlington, MA, 259-289.

Anderson, E. G., A. Davis-Blake, G. G. Parker. 2004 Managing outsourced product design: The effectiveness of alternative integration mechanisms. Presented at the 2004 New Orleans, Academy of Management Conference, Louisiana.

Anderson, E. G., N. Joglekar. 2005. A hierarchical modeling framework for product development planning. Prod. Oper. Manag. 14(3): 344-361.

Anderson, E. G., G. G. Parker. 2002. The effect of learning on the make/buy decision. Prod. Oper. Manag. 11(3): 313-339.

Armstrong, D. J., P. Cole. 1998. Managing distances and differences in geographically distributed work groups. P. Hinds, S. Kiesler, eds. Distributed Work. MIT Press, Cambridge, MA, 167-186.

Audretsch, D. B. 2003. Managing knowledge spillovers: The role of geographic proximity. J. A. C. Baum, O. Sorenson, eds. Geography and Strategy, Vol. 20. Elsevier, Amsterdam, $23-48$.

Baldwin, C. Y., K. B. Clark. 2000. Design Rules: The Power of Modularity. MIT Press, Cambridge, MA.
Bell, G. G., A. Zaheer 2007. Geography, networks, and knowledge flow. Organ. Sci. 18(6): 955-972.

Bennett, J. 2008. Ford revamps engineering centers to speed up product development. The Wall Street Journal. Available at http://online.wsj.com/article/SB120714646482283383.html (accessed date December 12, 2009).

Borgatti, S. P., M. G. Everett, L. C. Freeman. 2002. Ucinet for Windows: Software for Social Network Analysis. Analytic Technologies, Harvard, MA.

Brockhoff, K. 1998. Internationalization of Research and Development. Springer, Berlin.

Brown, S. L., K. M. Eisenhardt. 1995. Product development: Past research, present findings, and future directions. Acad. Manag. Rev. 20(2): 343-378.

Clark, K. B., T. Fujimoto. 1991. Product Development Performance: Strategy, Organization, and Management in the World Auto Industry. Harvard Business School Press, Boston, MA.

Cummings, J. N. 2004. Work groups, structural diversity, and knowledge sharing in a global organization. Manage. Sci. 50(3): 352-364.

Daft, R. L., R. H. Lengel. 1986. Organizational information requirements, media richness and structural design. Manage. Sci. 32(5): 554-571.

Dyer, J. H., H. Singh. 1998. The relational view: Cooperative strategy and sources of interorganizational competitive advantage. Acad. Manag. Rev. 23(4): 660-679.

Eppinger, S. D., A. R. Chitkara. 2006. The new practice of global product development. MIT Sloan Manag. Rev. 47(4): 22-30.

Eppinger, S. D., D. E. Whitney, R. P. Smith, D. A. Gebala 1994. A model-based method for organizing tasks in product development. Res. Eng. Design. 6(1): 1-13.

Espinosa, J. A., S. A. Slaughter, R. E. Kraut, J. D. Herbsleb. 2007. Familiarity, complexity, and team performance in geographically distributed software development. Organ. Sci. 18(4): 613630 .

Ethiraj, S. K., D. Levinthal. 2004. Modularity and innovation in complex systems. Manage. Sci. 50(2): 157-173.

Fuggetta, A. 2003. Open source software-An evaluation. J. Syst. Softw. 66: 77-90.

Gerwin, D., N. J. Barrowman. 2002. An evaluation of research on integrated product development. Manage. Sci. 48(7): 938952.

Gokpinar, B., W. J. Hopp, S. M. R. Iravani. 2010. The impact of misalignment of organizational structure and product architecture on quality in complex product development. Manage. Sci. 56(3): 468-484.

Greene, W. H. 2008. Econometric Analysis, 6th edn. Prentice Hall, Upper Saddle River, NJ.

Haas, M. R. 2006. Knowledge gathering, team capabilities, and project performance in challenging work environments. Manage. Sci. 52(8): 1170-1184.

Hansen, M. T., B. Lovas. 2004. How do multinational companies leverage technological competencies? Moving from single to interdependent explanations. Strateg. Manag. J. 25(8-9): 801822.

Harvey, M. G., D. A. Griffith. 2007. The role of globalization, time acceleration, and virtual global teams in fostering successful global product launches. J. Prod. Innov. Manag. 24: 486-501.

Hausman, J. A. 1978. Specification tests in econometrics. Econometrica 46: 1251-1271.

Henderson, R. M., K. B. Clark. 1990. Architectural innovation: The reconfiguration of existing product technologies and the failure of established firms. Admin. Sci. Q. 35: 9-30. 
Herbsleb, J. D., A. Mockus. 2003. An empirical study of speed and communication in globally distributed software development. IEEE Trans. Softw. Eng. 29(3): 1-14.

Hiltzik, M. 2011. 787 dreamliner teaches Boeing costly lesson on outsourcing. Los Angeles Times. Available at: http://articles.latimes.com/2011/feb/15/business/la-fi-hiltzik-20110215 (accessed date March 20, 2011).

Hinds, P., D. Bailey. 2003. Out of sight, out of sync: Understanding conflict in distributed teams: An empirical investigation. Organ. Sci. 14(6): 615-632.

Hinds, P., S. Kiesler. 1998. Distributed Work. MIT Press, Cambridge, MA.

Hinds, P., S. Kiesler. 2005. Understanding conflict in geographically distributed teams: The moderating effects of shared identity, shared context, and spontaneous communication. Organ. Sci. 16: 290-307.

Hoegl, M., H. Ernst, L. Proserpio. 2007. How teamwork matters more as team member dispersion increases. J. Prod. Innov. Manag. 24: 156-165.

Hoetker, G. 2007. The use of logit and probit models in strategic management research: Critical issues. Strateg. Manag. J. 28(4): 331-343.

Huang, G. Q., K. L. Mak. 1999. Current practices of engineering change management in UK manufacturing industries. Int. J. Oper. Prod. Manag. 19(1), 21-37.

Jarillo, J. C. 1988. On strategic networks. Strateg. Manag. J. 9: 31-41.

Jarvenpaa, S., D. E. Leidner. 1999. Communication and trust in global virtual teams. Organ. Sci. 19: 791-815.

Johnston, W. B. 1991. Global work force 2000: The new world labor market. Harvard Bus. Rev. 69(2): 115-127.

Kraut, R. E., S. R. Fussell, S. E. Brennan, J. Siegel. 1998. Understanding effects of proximity on collaboration: Implications for technologies to support remote collaborative work. P. Hinds, S. Kiesler, eds. Distributed Work. MIT Press, Cambridge, MA, 137-162.

Krishnan, V., C. Loch. 2005. A retrospective look at production and operations management articles on new product development. Prod. Oper. Manag. 14(4): 433-441.

Latane, B., J. H. Liu, A. Nowak, M. Bonevento, L. Zheng. 1995. Distance matters: Physical space and social impact. Personal. Soc. Psychol. Bull. 21(8): 795-805.

Leonardi, P. M., D. E. Bailey. 2008. Transformational technologies and the creation of new work practices: Making implicit knowledge explicit in task-based offshoring. MIS Q. 32(2): 411-436.

Loch, C. H., C. Terwiesch. 1999. Accelerating the process of engineering change orders: Capacity and congestion effects. J. Prod. Innov. Manag. 16(2): 145-159.

MacCormack, A. D. 2001. Product-development practices that work: How internet companies build software. MIT Sloan Manag. Rev. 42(2): 75-84.

MacCormack, A. D., C. Y. Baldwin, J. Rusnak. 2006. Exploring the structure of complex software designs: An empirical study of open source and proprietary code. Manage. Sci. 52(7): 1015-1030.

McDonough III, E. F., K. B. Kahn, G. Barczak. 2001. An investigation of the use of global, virtual, and colocated new product development team. J. Prod. Innov. Manag. 18: 110-120.

Metters, R. 2008. A typology of offshoring and outsourcing in electronically transmitted services. J. Oper. Manag. 26: 198-211.

Novak, S., S. D. Eppinger. 2001. Product complexity and the supply chain. Manage. Sci. 47(1): 189-204.
Parker, G. G., E. G. Anderson. 2002. From buyer to integrator: The transformation of the supply-chain manager in the vertically disintegrating firm. Prod. Oper. Manag. 11(1): 75-91.

Ray, S. 2011. Boeing may increase dividend as dreamliner deliveries start. Bloomberg. Available at: http://www.bloomberg.com/ news/2011-03-23/boeing-considers-buying-back- shares-boosting-dividend-in-2012.html (accessed date: March 23, 2011).

Sanchez, R., J. T. Mahoney. 1996. Modularity, felixibility and knowledge management in product and organization design. Strateg. Manag. J. 17: 63-76.

Saxenian, A. 1994. Regional Advantage: Culture and Competition in Silicon Valley and Route 128. Harvard University Press, Cambridge, MA.

Schilling, M., K. H. Steensma. 2001. The use of modular organizational forms: An industry-level analysis. Acad. Manag. J. 44(6): 1149-1168.

Soble, J. 2010. Nintendo delays launch of $3 \mathrm{~d}$ console. Financial Times. Available at: http://www.ft.com/cms/s/2/e0e2fbd8cbb0-11df-a4f5-00144feab49a.html\#axzz1PA6CXx4S (accessed date December 11, 2010).

Sosa, M. E., S. Eppinger, M. Pich, D. G. McKendrick, S. K. Stout. 2002. Factors that influence technical communication in distributed product development: An empirical study in the telecommunications industry. IEEE Trans. Eng. Manag. 49(1): $45-58$.

Stackpole, B. 2007. Boeing's global collaboration environment pioneers groundbreaking 787 dreamliner development effort. Design News. Available at: http://www.designnews.com/article/13616-Boeing's_Global_Collaboration_Environment_Pioneers_ Groundbreaking_787_Dreamliner_Development_Effort.php (accessed date January 15, 2008).

Stalk, G., T. M. Hout. 1990. Competing Against Time. Free Press, London.

Subramaniam, M. 2006. Integrating cross-border knowledge for transnational new product development. J. Prod. Innov. Manag. 23: 541-555.

Terwiesch, C., C. H. Loch. 1999. Managing the process of engineering change orders: The case of the climate control system in automobile development. J. Prod. Innov. Manag. 16(2): 160172.

Ulrich, K. 1995. The role of product architecture in the manufacturing firm. Res. Policy 24: 419-441.

Ulrich, K. T., D. J. Ellison. 2005. Beyond make-buy: Internalization and integration of design and production. Prod. Oper. Manag. 14(3): 315-330.

Ulrich, K. T., S. Pearson. 1998. Assessing the importance of design through product archeology. Manage. Sci. 44(3): 352-369.

Venkatraman, N. 2004. Offshoring without guilt. MIT Sloan Manage. Rev. 45(3): 14-16.

von Hippel, E. 1994. Sticky information and the locus of problem solving: Implications for innovation. Manage. Sci. 40(4): 429439.

von Hippel, E. 1998. The Sources of Innovation. Oxford University Press, New York.

Wasserman, S., K. Faust. 1994. Social Network Analysis. Cambridge University Press, London.

White, H. 1980. A heteroscedasticity-consistent covariance matrix estimator and a direct test for heteroscedasticity. Econometrica 48: 817-838. 